\title{
Network Pharmacology and Molecular Docking Reveal the Mechanism of Isodon Ternifolius (D. Don) Kudo Against Liver Fibrosis
}

\section{Le Qin}

GuangXi University of Chinese Medicine https://orcid.org/0000-0003-1517-8867

\section{Zhipin Zhou ( $\sim$ gxzhouzhipin@hotmail.com )}

Liuzhou People's Hospital of Guangxi Medical University https://orcid.org/0000-0002-3185-1161

\section{Hui Huang}

GuangXi University of Chinese Medicine

Jingjing Wang

GuangXi University of Chinese Medicine

\section{Yong Chen}

GuangXi University of Chinese Medicine

\section{Runan Nong}

GuangXi University of Chinese Medicine

\section{Research}

Keywords: Chines Medicine, Isodon ternifolius (D. Don), network pharmacology, molecular docking, liver fibrosis

Posted Date: November 30th, 2021

DOI: https://doi.org/10.21203/rs.3.rs-1088473/v1

License: (c) (i) This work is licensed under a Creative Commons Attribution 4.0 International License. Read Full License 


\section{Abstract}

\section{Background}

Isodon ternifolius (SanYe Xiang ChaCai, SYXCC) is a traditional Chinses medicine commonly used in the treatment of chronic hepatitis B in China. Many studies have demonstrated the hepatoprotective or antifibrotic effects of $S Y X C C$, but its pharmacological basis and mechanism remain unclear. In this study, we used in vitro models to validate the predicted results and reveal the potential mechanism of action and active ingredients with the help of network pharmacology methods and molecular docking.

Methods

We obtained the chemical structures of SYXCC by literatures. Potential targets of SYXCC were predicted by Swiss Target Prediction. The disease targets were collected through the databases of Gene Card. PPI protein interaction network was obtained using STRING database. Signaling pathway enrichment analysis was performed on drug-disease targets with of DAVID database. In vitro, human umbilical vein endothelial cell was used to validate the results predicted by network pharmacology.

Results

90 pathways related to liver fibrosis were obtained by KEGG enrichment. We were interested in the top 25 core target proteins involved in the TLR4, MAPK and PI3K-Akt signaling pathways associated with liver fibrosis. RT-PCR result showed that SYXCC could reduce EGFR, ERK1, Akt1, VEFGR-2, ERK2 mRNA expression.

\section{Conclusion}

In the study, our results suggest that a multitude of active ingredients of Isodon ternifouis play an important role in the liver fibrosis disease network. Inhibition of angiogenesis was a new potential pharmacological mechanism for the anti-hepatic fibrosis activity of Isodon ternifoius.

\section{Introduction}

Liver fibrosis (LF) is a reversible damage repair response, which is characterized by the accumulation of extracellular matrix (ECM). If the injury was acute or self-limiting, these changes were transient and the liver structure could return to its normal composition. However, if the insult is sustained, the liver parenchyma is replaced by fibrotic scar tissue, a process in which ECM and MMPs have recognized key functions ${ }^{[1]}$. Chronic liver diseases (CLDs) are characterized by long-term chronic hepatic parenchymal cell injury, a persistent inflammation response and a sustained activation of LF and damage repair response, so LF is present in most chronic liver diseases ${ }^{[2]}$. The prevalence and mortality of CLDs could be compared with major publish healthy issue related to common chronic diseases, such as diabetes, tuberculosis, and cardiovascular diseases. Previous studies have shown that 844 million people worldwide suffer from CLDs, with 2 million deaths per year ${ }^{[3]}$. Currently, curing LF remains a huge 
challenge globally, as drug treatment strategies can only slow down the process of LF, but not cure it. Therefore, it is necessary to explore drugs for the treatment of LF.

Traditional Chinese medicine (TCM) has a long history of preventing and treating diseases. TCM has curative effects in the treatment of liver diseases, especially in the field of anti-fibrosis, which has significant advantages and great development value. SYXCC, also known as the San Jie Mei in the Chinese folk ${ }^{[4]}$. The genus of SYXCC is Lamiaceae, whice is sweet, slightly bitter, and cool. It has the effects of clearing heat and detoxification, promoting dampness and soothing the liver ${ }^{[4]}$. It is used for treat enteritis, acute and chronic hepatitis and early cirrhosis of the liver ${ }^{[5]}$. Chinese patent medicine containing SYXCC, "Fufang sanyexiangchacai Pian", were used to treat acute and chronic hepatitis $\mathrm{B}^{[6,7]}$. Previous studies have found that $S Y X C C$ has anti-LF effect, the mechanism may be through inhibiting the

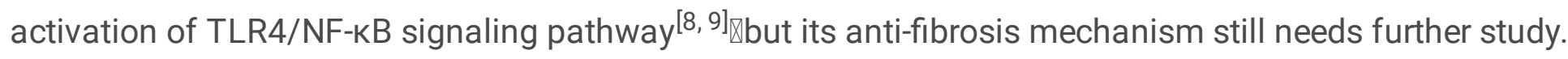

\section{Network pharmacology and molecular docking}

\subsection{The source of chemical constituents and the prediction of drug targets in SYXCC}

We searched the herbal databases from TCMSP (Traditional Chinese Medicine Database and Analysis Platform) (https://tcmsp-e.com/), ETCM (The Encyclopedia of Traditional Chinese Medicine) (http://www.tcmip.cn/ETCM/), and TCMID (Traditional Chinese Medicines Integrated Database) (http://47.100.169.139:8000/tcmid/) for hand-collected data on the ingredients of SYXCC. If no, we have collected the ingredients of SYXCC through literature search. The compound structures were drawn using ChemBioDraw Utra 14.0, saved in the mol2 format and converted into 3D strutures by ChemBio3D Ultra 14.0, and saved mol2 format file after miniming MM2 energy. Subsequently, with the help of Swiss Target Prediction (STP) (http://swisstargetprediction.ch/) database to predict the drug target, and get the drug target genes.

\subsection{Target genes of LF}

GenCard integrates gene-centric data from 150 network sources, including genomics, transcriptomics, proteomics, genetics, clinical and functional information. Liver fibrosis target genes were obtained from GenCard database(https://www.genecards.org/).

\subsection{Network construction}

\subsubsection{Compound-disease target network}

The online tool Venny2.1 (https://bioinfogp.cnb.csic.es/tools/venny/ ) was used to draw Venn diagrams to obtain target genes of compound-disease intersection. The visualization software Cytoscape3.7.1 was used to construct the compound-disease target network.

\subsubsection{Construction of Protein-protein Interaction (PPI) Network and Core Target Screenig}


The common target of SYXCC of compound-LF was imported into String database (https://string$\mathrm{db}$. org/), and Homo sapiens was selected. Finally, the PPI network is constructed, and the network is constructed, and the results were exported and saved as files.

\subsubsection{GO (Gene Ontology) and KEGG (Kyoto Encyclopedia of Genes and Genomes) enrichment analysis and construction of core target pathways}

The compound of SYXCC-LF common target genes were enriched by GO function and analyzed by KEGG pathway through the DAVID6.8(https://david.ncifcrf.gov/) biological information annotation database. GO enrichment analysis includes Molecular Function (MF), Cellular Component (CC), and Biological Process (BC). P.Value and FDR have been used to assess the correlation, set the parameters P.Value区 $0.05 \bowtie F D R \bowtie 0.05$. The core target was imported into KEGG enrichment and the relevant enrichment pathway was obtained through the website (https://www.genome.jp/kegg/tool/map_pathway1.html).

\subsection{Molecular docking}

Autodock 4.2 is an automated program for predicting the interaction between ligands and biological macromolecular targets, which is used in drug research and development ${ }^{[10]}$. We obtained the main enrichment pathways through enrichment analysis of the KEGG pathway, and used Autodock 4.2 to verify the molecular docking of the core targets related to the middle value of the protein interaction network with the chemical components of Isodon ternifoius.

\subsubsection{Acquisition and processing of target protein}

The 3D structure of candidate target is downloaded in the PDB protein database (https://www.rcsb.org/), and the result was saved in the pdb format, and then the visualization software PyMOL (TM) Molecular Graphics System, Version 2.4 was used to remove the water in the target protein Molecule and separation of small molecules.

\subsubsection{Docking steps and result evaluation}

(1) Ligand and receptor file preparation before docking: Small molecule ligand compounds and core proteins are imported into AutoDockTools (v1.5.6) for hydrogenation, charge calculation, charge distribution and save as "pdbq"; (2) Grid file preparation: After opening the Grid option to import the receptor and ligand, select the Grid Box, adjust the center of the box to wrap the macromolecular protein, and save the output as "gpf"; (3) Parameter file preparation: Open Docking to import receptor and ligand files, set the parameters to default values, and operate as follows: Docking/Search Parameters/Genetic Algorithm Parameters, Docking/Docking Parameters, Output/Genetic Algorithm output is saved as "dpf"; (4) Put the prepared protein files, compound files and corresponding folders into the corresponding folders, and use AutoDock (v1.5.6) for molecular docking; (5) Evaluation of results: The docking possibility and stability are evaluated by the spatial position and binding energy after docking. We choose the binding energy $\leq-5.0$ as the evaluation of receptor-ligand binding ${ }^{[11]}$. 


\section{Network Pharmacology And Molecular Docking Results}

\subsection{Compound of SYXCC}

Ethyl acetate extraction and n-butanol extraction from the roots of SYXCC were isolated and identified 25 compounds, following 3-carboxy formate-6,7-dihydroxy-1-(3',4'-dihydroxyphenyl)-naphthalene (1); 3carboxy-6,7-dihydroxy-1-(3',4'-dihydroxyphenyl)-naphthalene (2); 1-acetatexyl-2e,6e-dipiperonyl-3,7dioxabcyclo[3,3,0]-octane (3); 2-(3,4,5-trimethoxyphenyl)-6-(4-hydroxy-3-meth-oxyphenyl)-3,7-

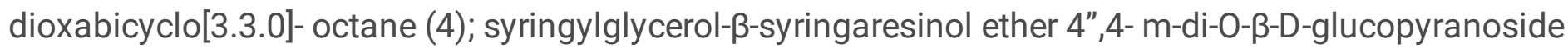
(5); clemastanin A (6); 3-(3,4-dihydroxyphenyl)acrylic acid 1-(3,4-dihydroxy-phenyl)-2-methoxycarbonylethyl ester (7); oxyneolignans A (8); 19-hydroxytotarol (9); (-)-lambertic acid (10); isodoternifolin B (11); longikaurin A (12); maoyecrystal F(13); 2a-hydroxy oleanolic acid (14); tormentic acid (15); myricetin 3neohesperidoside(16); 3,4-dimethoxy-1-hydroxylphenyl-O- $\beta$-D-glucopyranoside(17); 2,6-dimethoxy-phydroquinone1-O-B-D-glucopyranoside(18); 3,4,a-trihydroxyl-methyl phenylpropionate (19); erythro-I-csyringyl-glycerol (20); E-P-hydroxyl ethyl cinnamate(21); caffeic acid (22); 3, 4-dihydroxyphenacyl alco-hol (23); protocatechuic acid (24); Syringic acid (25) (The constituents of Isodon ternifolius (D.Don) Kudo were deried from Wang. K's master's thesis in Guangxi Normal University, 2018.)

\subsection{Network construction of prediction targets and disease targets of SYXCC}

The Swiss Target Prediction platform database was used to predict the target of SYXCC. Compounds 13, 19,20 , and 23 were eliminated due to no prediction results, and the remaining 21 compounds were predicted to have 395 targets (Probability $₫ 0$, delete duplicate targets). Using"liver fibrosis"as the search term, GeneCards retrieved $3794 \mathrm{LF}$ targets genes. The common target of compounds-hepatic fibrosis was imported into Cytoscape 3.7.2 for network visualization (Figure 2). This network has a total of 319 nodes and 626 edges.

\subsection{Target protein interaction network construction and core target screening}

The 298 predicted targets of SYXCC for the treatment of LF were imported into the STRING datebase (https://string-db.org/), and then the protein interaction network was constructed. The target point is hidded without network interaction, and other parameters are the default values. (Fig 3).

\subsection{Screening GO function enrichment and KEGG signal pathway analysis}

In order to explain the possible role of the candidate targets, the targets were used for GO function enrichment and KEGG pathway analysis through the DAVID6.8 database. GO enrichment analysis yielded a total of 219 data results. 23 results are used to describe the location of gene products in cells, such as cellular components (CC) such as endoplasmic reticulum, mitochondria, or nucleus. 33 results were used to describe the functions of individual gene products, such as molecular functions (MF) such as binding activity and catalytic activity. 164 results are used to describe an orderly biological process (BP) with multiple steps, such as cell growth, proliferation, apoptosis, and signal transduction. 


\subsection{Molecular docking}

Due to the drug targets of SYXCC are mainly enriched in the MAPK signaling pathway and the PI3K-Akt signaling pathway (Figure 7), the core targets of this study are TNF (PDB ID:1EXT), Akt1(PDB ID:4EKL), MAPK1(PDB ID:6GLD), EGFR (PDB ID:5d41), TLR4(PDB ID:2z65).

\section{Experiment}

\subsection{Materials \& Methods}

\subsubsection{Medicinal materials preparation}

The dried roots of SYXCC were purchased from Guangxi xianju Chinese Medicine Technology Company (20190501, Guangxi Province, China) in October 2019. The SYXCC of rhizome was soaked in 10 times waters for 30 minutes, then boiled for 60 minutes. Gauze was used to filter the herb residue and the filtrate was collected. The above process was repeated three times.95\% ethanol was used for extraction. After standing for 24 hours, the sediment was filtered off and the filtrate was collected. The filtrate was concentrated into an extract and refrigerated at $-80^{\circ} \mathrm{C}$. SYXCC extract was prepared with DMEM solution and used after filtration.

\subsubsection{Cell culture}

Human umbilical vein endothelial cells (HUVECs) were Cell Bank of Typ Culture Collection of Chinese Academy of Sciences (Shanghai, China). HUVECs were cultured in DMEM complete medium (GIBCO, cat \# 11965092) containing $10 \%$ fetal bovine serum FBS (GIBCO, cat \# 10099-141) in $5 \% \mathrm{CO} 2,37^{\circ} \mathrm{C}, 100 \%$ saturated humidity $\mathrm{CO} 2$ incubator.

\subsubsection{MTT assay}

HUVECs were seeded in 96 well plates with $1 \times 10^{4}$ cells/well and cultured in DMEM medium containing different concentrations $(2 \mathrm{ug} / \mathrm{ml}, 5 \mathrm{ug} / \mathrm{ml}, 10 \mathrm{ug} / \mathrm{ml}, 40 \mathrm{ug} / \mathrm{ml}, 75 \mathrm{ug} / \mathrm{ml})$ of $S Y X C C$ extract for $24 \mathrm{~h}$. Each group was repeated three times. MTT cell proliferation and cytotoxicity kit (China, Solarbio, cat\# M1020) was used to detect cell viability and proliferation inhibition rate. After $24 \mathrm{~h}$ of culture, $20 \mu \mathrm{L}$ of MTT (5 $\mathrm{mg} / \mathrm{mL}$ ) was added to each well. After $4 \mathrm{~h}$ culture, the supernatant was centrifuged, and $150 \mu \mathrm{L}$ of DMSO was added to each well to dissolve the crystalline particles. The absorbance was measured at $570 \mathrm{~nm}$.

\subsubsection{Scratch healing assay}

Scratch healing assay used to assess changes in migration of HUVECs. Differnet groups of cells were seeded into 6-well plates. After the cells have grown to full, the central portion of the cells was removed by scoring the central area of cell growth with tip, and then the cells continue to be cultured with a culture medium containing different concentrations of SYXCC. After 24 hours, the cells were observed under 
microscope whether migrated to the central scratch area, and photographed. Image $\mathrm{J}$ software was used to analyze migration and calculate migration rate.

\subsubsection{Tube formation assay}

To observe microvasculature, HUVECs treated by different concentration were seeded in 96-well plates with matrix adhesive with substrate gel (Solarbio, cat\# 356234 ) and cultured at $37^{\circ} \mathrm{C}$ in a $5 \% \mathrm{CO} 2$ incubator. After 6 hours of culture, the vascular network was imaged by inverted microscope at 10x and vascular network was quantified by Image J's package with angiogenesis analyzer.

\subsubsection{Quantitative Real-Time Polymerase Chain Reaction (PCR)}

Total RNA was extracted by TriQuick Reagent Total RNA Extraction Kit (Solarbio, R1100) and stored at-80 ${ }^{\circ} \mathrm{C}$. RNA was reverse transcribed to cDNA using PrimeScript ${ }^{\mathrm{TM}} \mathrm{RT}$ reagent Kit with gDNA Eraser (Perfect Real Time) (Takara, RR036A) according to the instructions and stored at $-80^{\circ} \mathrm{C}$. The predicted target genes EGFR, VEGFR-1, ERK1, ERK2, Akt mRNA were amplified by Veriti ${ }^{\mathrm{TM}}$ 96-Well Thermal Cycler Applied Biosystems $\circledast$ in Real-Time PCR System (Agilen, AriaMx). PCR conditions: protein denaturation $95^{\circ} \mathrm{C}$ for $30 \mathrm{~s}, 40$ cycles, each cycle $95^{\circ} \mathrm{C}$ for $5 \mathrm{~s}, 60^{\circ} \mathrm{C}$ for $10 \mathrm{~s}, 72{ }^{\circ} \mathrm{C}$ for $30 \mathrm{~s}$. $\beta$-ACTIN was used as the internal reference to correct the target $\mathrm{Ct}$ value, and $2^{-\triangle \Delta}$ was used to calculate the relative expression of mRNA. All primers were synthesized by Sangon Biotech in Shanghai (Table1).

Table 1 mRNA primer

\begin{tabular}{|lllll|}
\hline Gen & Gen bank & primer & Sequence $\left(5^{\prime}\right.$->3') & Length \\
EGFR & NM_001346897.2 & $\mathrm{F}$ & GTCTCCAGTGTCCAAGCCAG & 20 \\
\cline { 3 - 5 } VEGFR-2 & NM_002253.4 & $\mathrm{F}$ & AGACCGGCTGAAGCTAGGTA & 20 \\
\cline { 3 - 6 } & & $\mathrm{R}$ & CGATGCTCACTGTGTGTTGC & 20 \\
\hline Akt1 & NM_001014431.2 & $\mathrm{F}$ & TCCCCCAGTTCTCCTACTCG & 20 \\
\cline { 3 - 6 } & & $\mathrm{R}$ & TCCCTCCAAGCTATCGTCCA & 20 \\
ERK1 & NM_001040056.3 & $\mathrm{F}$ & GACTCCAAAGCCCTTGACCT & 20 \\
\cline { 3 - 5 } & & $\mathrm{R}$ & GGACTGGCCCACCTCATC & 18 \\
\hline ERK2 & NM_002745.5 & $\mathrm{F}$ & TCGGTGTTCTTCTTCCCAGT & 20 \\
\cline { 3 - 5 } & & $\mathrm{R}$ & CGGCTCAAAGGAGTCAAAGTG & 21 \\
\hline
\end{tabular}

\subsubsection{Statistical analysis}

All data are presented as mean \pm standard deviation (SD) and were evaluated using one-way analysis variance. $p \rrbracket 0.05$ or $p \bigotimes 0.01$ was considered statistically significant. All statistical analyses were performed 
using SPSS 26.0 .

\subsection{Experimental results}

\subsubsection{Effects of SYXCC on proliferation of HUVCs in vitro.}

In aim of evaluating the effect of SYXCC extract on the proliferation viability of HUVECs, we experimented with a variety of concentrations of SYXCC extract $(2 \mu \mathrm{g} / \mathrm{ml}, 5 \mu \mathrm{g} / \mathrm{ml}, 15 \mu \mathrm{g} / \mathrm{ml}, 45 \mu \mathrm{g} / \mathrm{ml}, 75 \mu \mathrm{g} / \mathrm{ml})$ to treat HUVECs. MTT results showed that IC50 was $11.91 \mu \mathrm{g} / \mathrm{ml}$.

\subsubsection{SYXCC extract inhibits migration and angiogenesis of HUVCs in vitro}

Many evidences show that angiogenesis is involved in the development of liver fibrosis. Hypoxia and inflammation are the main triggers of angiogenesis. Fibroblasts were located at the intersection between angiogenesis and fibrosis, and play a key role in angiogenesis ${ }^{[13,14]}$. In the pharmacology network, we found that the mechanism of the anti-fibrotic of SYXCC may be related to angiogenesis. In vitro, we investigated whether SYXCC has inhibitory effect on angiogenesis. Scratch healing and tube formation assays demonstrate that SYXCC extracts inhibited the migration of HUVECs in a dose-dependent manner and significantly inhibited the formation of blood vessels.

\subsubsection{SYXCC extract inhibits activation of PI3K/Akt and ERK1/2 Signaling Pathways in Vitro}

We have predicted that multiple SYXCC ingredients were involved in the regulation of PI3K/Akt and ERK1/ERK2 signaling pathways via network pharmacology. It has been demonstrated that ERK1/2 and $\mathrm{PI} 3 \mathrm{~K} /$ Akt were important kinase cascade signaling pathways in vivo, regulating HSCs of activation, proliferation, differentiation or intrahepatic vessels, resulting in the progression of livre fibrosis ${ }^{[15-20]}$. Akt, also known as protein kinase B (PKB), was a serine/threonine protein kinase that plays an important role in the development of alcoholic hepatitis, including promoting cell proliferation, migration, and transcription, and at the same time damage apoptosis. It was found that Akt1 and Akt2 in the Akt family are important in the pathological development of alcoholic hepatitis, and the mechanism is to regulate the occurrence of inflammatory response and fibrosis in the progression of alcoholic hepatitis ${ }^{[21]}$. It was found that PI3K inhibitors could inhibit the activation of ERK and the expression of c-fos ${ }^{[22]}$.PIK3R1 or Akt3 mice or HSC cells were knocked out, the expression of a-SMA and collagen I are reduced, and the degree of LF is reduced ${ }^{[20]}$. As the main signal transduction pathway involved in the phenotypic response of liver myofibroblasts, the ERK signaling pathway plays an important role in the progression of LF ${ }^{[15]}$. When Raf kinase inhibitory protein (RKIP) was blocked in rat liver tissue and primary HSCs, the ERK signaling pathway was activated to promote the production of a-SMA, MMP-1, MMP-2 and collagen, aggravate $\mathrm{LF}^{[23]}$. It has been reported that when fed with choline deficiency and supplemented with ethionine diet, the degree of LF in ERK knockout mice was relieved, the expression of fibrosis maker aSMA protein was reduced, and the percentage of proliferation and activated T cells was reduced ${ }^{[17]}$. 
Epidermal growth factor receptor (EGFR) was a transmembrane receptor tyrosine kinase that was activated by variety of signaling pathways to mediate cell proliferation and differentiation ${ }^{[24]}$. EGFR was important in the progression of $L F^{[25,26]}$. EGFR was up-regulated in LF. EGFR was blocked, it could alleviate the progress of LF by reducing lipid deposition, anti-oxidative stress, inhibiting HSCs activation and the release of inflammatory factors ${ }^{[25-27]}$.VEGF was the most important pro-angiogenic cytokine and plays a central role in angiogenesis because it was related to all the steps of angiogenesis: increasing vascular permeability, inducing endothelial cell proliferation, and regulating the diameter of new blood vessel lumen ${ }^{[28]}$.VEGF signaling pathway was blocked, it could effectively inhibit angiogenesis ${ }^{[29,30]}$. VEGF plays an important role in the progression of LF, promoting the activation, proliferation and collagen production of HSC ${ }^{[31]}$. In the liver, VEGF and its receptor tyrosine kinases VEGFR1(FIt-1) and VEGFR2(FIt-2) were not only expressed in the endothelial cells and hepatocytes of the liver, but also expressed in mesenchymal cells, especially in activation of $\mathrm{HSC}^{[32,33]}$.

PCR suggested that SYXCC significantly inhibited the activation of PI3K, Akt, ERK1/2 and reduced ERFG, VEGFR-2 mRNA expression in vitro. The results showed that inhibition of angiogenesis by regulating $\mathrm{PI} 3 \mathrm{~K} / \mathrm{Akt}$ and ERK1/2 signaling pathways may be a new mechanism of anti-hepatic fibrosis.

\section{Discussion}

Network pharmacology found that the SYXCC through the MAPK pathway ${ }^{[34]}$, TNF pathway ${ }^{[35]}$, PI3K-Akt pathway ${ }^{[36]}$, TLR4/NF-KB pathway ${ }^{[37]}$, VEGF pathway ${ }^{[31]}$, HIF-1 ${ }^{[38]}$, which are involved in the regulation of viral infection, inflammatory response, angiogenesis, and initiate the progress of fibrosis. Our previous studies have shown that SYXCC has the effects of protecting the liver and anti-LF, could down-regulate the activation of the TLR4 signaling pathway, and reduce interleukin-1 $\beta(I L-1 \beta)$, interleukin-6(IL-6), tumor necrosis factor- $a(T N F-a)^{[8,9]}$ but its mechanism and pharmacological basis are still unclear. In this study, we used network pharmacology and molecular docking technology to revealed the mechanism and potential active ingredients of SYXCC against LF, and verified it through in vitro experiments. The extract of SYXCC could inhibit the formation of blood vessels, possibly by inhibiting ERK1/ERK2 signaling pathway and the PI3K/Akt signaling pathway, inhibiting angiogenesis with inhibiting the progression of LF.

More and more evidences show that angiogenesis plays a crucial role in the progression of LF. Hepatic angiogenesis occurs in CLDs by inflammation and progressive fibrosis ${ }^{[13]}$. HSC is the core of the pathogenesis of LF, and plays an important role among liver inflammation, fibrosis and angiogenesis. Quiescent HSCs could be stimulated by a variety of growth factors, pro-inflammatory mediators produced by damaged resident cell types, and hypoxia to promote the formation of new blood vessels, which in turn could become a bridge between acute and chronic inflammation ${ }^{[39]}$. Evidence shows that under clinical and experimental conditions, liver angiogenesis and fibrosis were closely related ${ }^{[36,38]}$. Hypoxia, inflammation and HSC were considered to be the main causes of angiogenesis. Pro-fibrosis cells were located at the intersection between inflammation, angiogenesis, and fibrosis, and also play a key role in 
angiogenesis $^{[13,14]}$. The TLR4 family is a type $\otimes$ transmembrane protein, which mediates the recognition of pathogen-related molecular patterns (PAMP) and damage-related molecular patterns (DAMP) from dead or damaged cells, initiates immune responses, and induces inflammation. It was found that LF is related to TLR4. TLR4 activates NF-KB, IRF-3, AP-1, etc., promotes the release of inflammatory factors and pro-fibrotic factors, and activates hepatic HSCs in the progress of LF. ${ }^{[40]}$. TNF binds to its ligands TNF receptor 1(TNFR1) and TNF receptor 2 (TNFR2) activates downstream NF-KB, MAPKs and Akt signaling pathways, and promotes the release of inflammatory factors ${ }^{[35]}$. Wree etc, found that TNF and IL-17 activate NLRP3 inflammasomes to amplify the inflammatory response in the progression of LF. ${ }^{[41]}$. We have previously confirmed that $S Y X C C$ could inhibit the activation of TLR4/NF-KB pathway and reduce the production of IL-6 and TNF-a in inhibiting the progression of $\mathrm{LF}^{[8,9]}$.

ERK1/2 and PI3K/Akt were regulated by a variety of cell growth factors, including VEGF, PDGF, EGF, etc. [42], and produce fibrosis through a variety of mechanisms, including promoting inflammation, activated endothelial cells releasing fibrosis enhancing molecules and activated $\operatorname{HSCs}^{[43,44]}$, up-regulate a-SMA, collagen, integrin, fibronectin, IFN-a, IL-6, IL-10 ${ }^{[31]}$. Once the liver was damaged, HSCs autocrine or paracrine cell growth factors such as VEGF, PDGF, EGF, which growth factors bind to the tyrosine kinase receptor on the cell membrane to transmit the activation signal to GTPase Ras, and then Ras is recruited to the plasma membrane and binds to Raf ${ }^{[48]}$. Ras was the common upstream molecule of ERK $1 / 2$ and $\mathrm{PI} 3 \mathrm{~K} /$ Akt signaling pathways ${ }^{[45]}$. The activation signal was phosphorylated through the Ras/Raf/MEK/ERK cascade, and then also phosphorylates the phosphatidylinositol 3-kinase-Akt-p70 S6 kinase (PI3K-Akt-p70 S6k) cascade, which phosphorylates its substrate and expresses various transcription factors involved in cell proliferation and differentiation, secrete pro-fibrosis factors and angiogenesis in the progress of $L F^{[15,46]}$. The PI3K/Akt signaling pathway regulated the synthesis of MMP and the degradation of extracellular matrix through the activation of HSCs in the progression of LF. Type I collagen was the main component of ECM and the main collagen secreted by activated HSC. PI3K regulated the transcriptional expression of collagen and plays an important regulatory role in the synthesis of collagen ${ }^{[47]}$.

In the drug-disease network, we found 3-carboxy formate-6,7-dihydroxy-1-(3',4'-dihydroxyphenyl)naphthalene (2), 19-hydroxytotarol (9), (-)-Lambertic acid (10), 2a-hydroxy oleanolic acid (14), tormentic acid (15), E-P-hydroxyl ethyl cinnamate (21), play an important regulatory role. Some compounds have been reported to have therapeutic effects on liver disease, such as ursolic acid and oleanolic acid. Oleanolic acid and ursolic acid were classified as pentacyclic triterpene acid compounds, which have been reported to treat acute and chronic liver diseases. Its mechanism includes anti-oxidative stress ${ }^{[48-51]}$, inhibition of HSC activation and reduction of the production of pro-fibrotic factors ${ }^{[52,53]}$, reduction of blood lipids, inhibition of liver lipid synthesis and promotion of lipid metabolism ${ }^{[54]}$, and improvement of intestinal flora ${ }^{[55,56]}$. Tormentic aicd could prevent LF by blocking PI3K/Akt/mTOR and NF-KB signaling pathways to inhibit HSC activation ${ }^{[57]}$. 19-hydroxytotarol could antioxidant by inhibiting mitochondrial lipid peroxidation ${ }^{[58]}$. 3-carboxy formate-6,7-dihydroxy-1-(3',4'-dihydroxyphenyl)-naphthalene (1) was first 
discovered as a component of SYXCC, which could relieve inflammation and reduce LPS-induced RAW264.7 cells secrete PGE2 and TNF-a ${ }^{[4]}$. The molecular docking results also showed that 2a-hydroxy oleanolic acid (14), tormentic acid (15), 1-acetylacetonyl-2e, 6e-dipiperonyl-3, 7-dioxabicyclo [3, 3, 0]octane (3), 19-hydroxytotarol (9), longikaurin A (12) could bind to the core target.

Network pharmacology is based on the theory of systems biology. It integrates the biological system network and the drug action network, and then analyzes the regulatory role of the drug in the disease network, emphasizing the overall regulatory role. The research method of network pharmacology is consistent with the multi-target and multi-path mechanism of TCM. Among them, 1-acetylacetonyl-2e-6edipiperonyl-3, 7-dioxabicyclo[3, 3, 0]-octane (3) has anti-inflammatory activity ${ }^{[12]}$. Iongikaurin A (12) activate the ROS/JNK/c-Jun signaling pathway by inhibiting the expression of Skp2, resulting in the induction of cycle arrest and apoptosis of liver cancer cells ${ }^{[59]}$. Particularly, (-)-lambertic acid was docked by multiple core target proteins, indicating that (-)-lambertic acid may be a potential active ingredient in SYXCC. It has been reported that (-)-lambertic acid exists in Lambertae plants such as Isodon macrophylla ${ }^{[60]}$, Isodon amethystoides ${ }^{[61]}$, which has anti-tumor ${ }^{[62]}$ and antioxidant effects ${ }^{[61]}$. However, there is still no report on the effect of (-)-lambertic acid on liver disease. In the future, we will further explore the effect and mechanism of (-)-lambertic acid on anti-liver and anti-fibrosis. We concluded that SYXCC inhibits LF through multiple compound and multiple drug targets.

\section{Conclusion}

Network pharmacology and molecular docking technology explored the mechanism of anti-hepatic fibrosis and potential active ingredients of SYXCC. The experiment verified that the anti-LF mechanism of the SYXCC might be the inhibition of intrahepatic blood vessels. We concluded that: (1) SYXCC multitarget regulation TLR4, PI3K/Akt, ERK1/ERK2, inhibits the synthesis and release of inflammatory cytokines, fibrosis-related factors, and inhibits the formation of blood vessels. (2) The most potential activities of SYXCC include 19-hydroxytotarol, (-)-lambertic acid, longikaurin A, 2a-hydroxy oleanolic acid, tormentic acid, which are easily combined with TLR4, TNF, MAPK, EGFR.

\section{Abbreviations}

LF: Liver Fibrosis; TCM: Traditional Chinese Medicine; TNF: Tumor Necrosis Factor; MAPK1: Mitogenactivated Protein Kinase 1; EGFR: Epidermal Growth Factor Receptor; TLR4: Toll-like Receptors; PPI: Protein Protein Interaction; ECM: Extracellular Matrix; CLDs: Chronic Liver Diseases; TCMSP: Traditional Chinese Medicine Database and Analysis Platform; ETCM: The Encyclopedia of Traditional Chinese Medicine; TCMID: Traditional Chinese Medicines Integrated Database; STP: Swiss TargetPrediction; GO: Gene Ontology; KEGG: Kyoto Encyclopedia of Genes and Genomes; MF: Molecular Function; CC: Cellular Component; BP Biological Process; SYXCC: SYXCC, HUVECs: Human Umbilical Vein Endothelial Cells; IL-13: Interleukin-13; IL-6: Interleukin-6; HSC: Hepatic Stellate Cells; PAMP: Pathogen-related 
Molecular Patterns; DAMP: Damage-related Molecular Patterns; TNFR1: TNF Receptor 1; TNFR2: TNF Receptor 2; PKB: Protein Kinase B; RKIP $\quad$ : Raf Kinase Inhibitory Protein.

\section{Declarations}

\section{Acknowledgements}

Not applicable

\section{Author's contributions}

Le Qin: methodology, software, Formal analysis, Writing - Original Draft; Zhipin Zhou: Project administration, Supervision, Conceptualization; Huang Hui: Writing - Review \& Editing, Formal analysis; Yong Chen: Investigation, Data Curation; Jingjing Wang: Visualization, Investigation; Runang Nong: Software, Writing - Review \& Editing.

\section{Funding}

This work was supported by the National Natural Science Foundation of China (No.81760751) and Liuzhou People's Hospital Introduces Top Talents Scientific Research Start-up Fund (No. LRYGCC202104) and the Guangxi Natural Science foundation (No. 2021 GXNSFAA075020).

\section{Authors statement}

Each of the coauthors has seen and agrees with each of the changes made to this manuscript in the revision and to the way his or her name is listed.

\section{Competing interests}

The authors declare that there are no conflicts of interest.

\section{References}

1. Hernandez-Gea V, Friedman SL. Pathogenesis of liver fibrosis [J]. Annu Rev Pathol, 2011, 6: 425-456. DOI: 10.1146/annurev-pathol-011110-130246.

2. Parola M, Pinzani M. Liver fibrosis: Pathophysiology, pathogenetic targets and clinical issues [J]. Mol Aspects Med, 2019, 65: 37-55. DOI: 10.1016/j.mam.2018.09.002.

3. Marcellin P, Kutala BK. Liver diseases: A major, neglected global public health problem requiring urgent actions and large-scale screening [J]. Liver Int, 2018, 38 Suppl 1: 2-6. DOI: 10.1111/liv.13682.

4. Zhang $\mathrm{Y}$, Wang $\mathrm{K}$, Chen $\mathrm{H}$, et al. Anti-inflammatory lignans and phenylethanoid glycosides from the root of SYXCC (D.Don) Kudô [J]. Phytochemistry, 2018, 153: 36-47. DOI: 10.1016/j.phytochem.2018.05.017. 
5. Wu ZY, Li XW. Flora Repubulicae Popularis Sinicae [J]. Science Press Beijing PP, 1977, 66: 436-439.

6. Liu M, Wang WG, Sun HD, et al. Diterpenoids from Isodon species: an update [J]. Nat Prod Rep, 2017, 34(9): 1090-1140.

7. Li R, Zhao LS, Xiong HC, et al.Observation on Curative Effect of Treatment of 98 Case of Chronic Hepatits B with Compound Rabdosia Ternifolia Table. Sichuan Med J. 2008, 29(4):397-398.

https://doi.org/10.16252/j.cnki.issn1004-0501-2008.04.004.

8. Zhou ZP, Xiao XQ, Wang Q, et al. Effect of Isodon ternifolius on NF-KB/IL-6 signaling pathway in CCl-4 liver fibrosis rats. Pharmacol Clin Chin Mater Med. 2016, 32(6):150-153.

https://doi.org/10.13412/j.cnki.zyyl.2016.06.040.

9. Zhou, ZP, Xiao XQ, Wang Q, et al. Effect of Herba Isodonis Ternifoliae on Toll-like Receptor 4 Pathway in Rats with Hepatic Fibrosis Induced by Carbon Tetrachloride. Tradit Chin Drug Res Clin Pharmacol. 2017, 28(2):183-186+262. https://doi.org/10.19378/j.issn.1003-9783.2017.02.008.

10. Harris R, Olson AJ, Goodsell DS. Automated prediction of ligand-binding sites in proteins [J]. Proteins, 2008, 70(4): 1506-1517.

11. Yuan WB, Wei YM, Chen Y, et al. Mechanisms of eleutheroside for treatment of diabetes mellitus type 2 based on network pharmacology. Acta Pharm Sin. 2019, 54(11):1982-1989.

https://doi.org/10.16438/j.0513-4870.2019-0350.

12. Elpek G. Angiogenesis and liver fibrosis [J]. World J Hepatol, 2015, 7(3): 377-391.

13. Lefere S, Devisscher L, Geerts A. Angiogenesis in the progression of non-alcoholic fatty liver disease [J]. Acta Gastroenterol Belg, 2020, 83(2): 301-307.

14. Foglia B, Cannito S, Bocca C, et al. ERK Pathway in Activated, Myofibroblast-Like, Hepatic Stellate Cells: A Critical Signaling Crossroad Sustaining Liver Fibrosis [J]. Int J Mol Sci, 2019, 20(11):2700.

15. Ge X, Guo R, Qiao Y, et al. The G protein-coupled receptor GPR30 mediates the nontranscriptional effect of estrogen on the activation of PI3K/Akt pathway in endometrial cancer cells [J]. Int J Gynecol Cancer, 2013, 23(1): 52-59.

16. Jeng KS, Lu SJ, Wang CH, et al. Liver Fibrosis and Inflammation under the Control of ERK2 [J]. Int J Mol Sci, 2020, 21(11):3796.

17. Lavoie H, Gagnon J, Therrien M. ERK signalling: a master regulator of cell behaviour, life and fate [J]. Nat Rev Mol Cell Biol, 2020, 21(10): 607-632.

18. Mannella P, Brinton RD. Estrogen receptor protein interaction with phosphatidylinositol 3-kinase leads to activation of phosphorylated Akt and extracellular signal-regulated kinase 1/2 in the same population 
of cortical neurons: a unified mechanism of estrogen action [J]. J Neurosci, 2006, 26(37): 9439-9447.

19. Wang J, Chu ES, Chen HY, et al. microRNA-29b prevents liver fibrosis by attenuating hepatic stellate cell activation and inducing apoptosis through targeting PI3K/AKT pathway [J]. Oncotarget, 2015, 6(9): 7325-7338.

20. Reyes-Gordillo K, Shah R, Arellanes-Robledo J, et al. Akt1 and Akt2 Isoforms Play Distinct Roles in Regulating the Development of Inflammation and Fibrosis Associated with Alcoholic Liver Disease [J]. Cells, 2019, 8(11):1337.

21. Marra F, Pinzani M, Defranco R, et al. Involvement of phosphatidylinositol 3-kinase in the activation of extracellular signal-regulated kinase by PDGF in hepatic stellate cells [J]. FEBS Lett, 1995, 376(3): 141145.

22. Huang Q, Liang C, Wei L, et al. Raf Kinase Inhibitory Protein Down-Expression Exacerbates Hepatic Fibrosis In Vivo and In Vitro [J]. Cell Physiol Biochem, 2016, 40(1-2): 49-61.

23. Komposch K, Sibilia M. EGFR Signaling in Liver Diseases [J]. Int J Mol Sci, 2015, 17(1):30.

24. Fuchs BC, Hoshida Y, Fujii T, et al. Epidermal growth factor receptor inhibition attenuates liver fibrosis and development of hepatocellular carcinoma [J]. Hepatology, 2014, 59(4): 1577-1590.

25. Liang D, Chen $\mathrm{H}$, Zhao $\mathrm{L}$, et al. Inhibition of EGFR attenuates fibrosis and stellate cell activation in dietinduced model of nonalcoholic fatty liver disease [J]. Biochim Biophys Acta Mol Basis Dis, 2018, 1864(1): 133-142.

26. Qian Y, Han J, Zhou L, et al. Inhibition of Epidermal Growth Factor Receptor (EGFR) Reduces Lipopolysaccharide (LPS)-Induced Activation and Inflammatory Cytokines in Hepatic Stellate Cells In Vitro [J]. Med Sci Monit, 2018, 24: 5533-5541. DOI: 10.12659/MSM.909901.

27. Carmeliet P, Jain RK. Molecular mechanisms and clinical applications of angiogenesis [J]. Nature, 2011, 473(7347): 298-307.

28. Ding $Q$, Tian XG, Li Y, et al. Carvedilol may attenuate liver cirrhosis by inhibiting angiogenesis through the VEGF-Src-ERK signaling pathway [J]. World J Gastroenterol, 2015, 21(32): 9566-9576.

29. Park S, Kim JW, Kim JH, et al. Differential Roles of Angiogenesis in the Induction of Fibrogenesis and the Resolution of Fibrosis in Liver [J]. Biol Pharm Bull, 2015, 38(7): 980-985.

30. Luo J, Liang Y, Kong F, et al. Vascular endothelial growth factor promotes the activation of hepatic stellate cells in chronic schistosomiasis [J]. Immunol Cell Biol, 2017, 95(4): 399-407.

31. Ankoma-Sey V, Matli M, Chang KB, et al. Coordinated induction of VEGF receptors in mesenchymal cell types during rat hepatic wound healing [J]. Oncogene, 1998, 17(1): 115-121. 
32. Ishikawa K, Mochida S, Mashiba S, et al. Expressions of vascular endothelial growth factor in nonparenchymal as well as parenchymal cells in rat liver after necrosis [J]. Biochem Biophys Res Commun, 1999, 254(3): 587-593.

33. Shen X, Guo H, Xu J, et al. Inhibition of IncRNA HULC improves hepatic fibrosis and hepatocyte apoptosis by inhibiting the MAPK signaling pathway in rats with nonalcoholic fatty liver disease [J]. J Cell Physiol, 2019, 234(10): 18169-18179.

34. Kalliolias GD, Ivashkiv LB. TNF biology, pathogenic mechanisms and emerging therapeutic strategies [J]. Nat Rev Rheumatol, 2016, 12(1): 49-62.

35. Gong Z, Lin J, Zheng J, et al. Dahuang Zhechong pill attenuates CCl4-induced rat liver fibrosis via the PI3K-Akt signaling pathway [J]. J Cell Biochem, 2020, 121(2): 1431-1440.

36. Lai L, Chen $Y$, Tian $X$, et al. Artesunate alleviates hepatic fibrosis induced by multiple pathogenic factors and inflammation through the inhibition of LPS/TLR4/NF-KB signaling pathway in rats [J]. Eur $\mathrm{J}$ Pharmacol, 2015, 765: 234-241.

37. Ju C, Colgan SP, Eltzschig HK. Hypoxia-inducible factors as molecular targets for liver diseases [J]. J Mol Med (Berl), 2016, 94(6): 613-627.

38. Zadorozhna M, Di Gioia S, Conese M, et al. Neovascularization is a key feature of liver fibrosis progression: anti-angiogenesis as an innovative way of liver fibrosis treatment [J]. Mol Biol Rep, 2020, 47(3): 2279-2288.

39. Pradere JP, Troeger JS, Dapito DH, et al. Toll-like receptor 4 and hepatic fibrogenesis [J]. Semin Liver Dis, 2010, 30(3): 232-244.

40. Wree A, Mcgeough MD, Inzaugarat ME, et al. NLRP3 inflammasome driven liver injury and fibrosis: Roles of IL-17 and TNF in mice [J]. Hepatology, 2018, 67(2): 736-749.

41. Karaman S, Leppänen VM, Alitalo K. Vascular endothelial growth factor signaling in development and disease [J]. Development, 2018, 145(14): dev151019.

42. Sahin H, Borkham-Kamphorst E, Kuppe $\mathrm{C}$, et al. Chemokine Cxcl9 attenuates liver fibrosis-associated angiogenesis in mice [J]. Hepatology, 2012, 55(5): 1610-1619.

43. Yoshiji $\mathrm{H}$, Kuriyama S, Yoshii J, et al. Vascular endothelial growth factor and receptor interaction is a prerequisite for murine hepatic fibrogenesis [J]. Gut, 2003, 52(9): 1347-1354.

44. Castellano E, Downward J. Role of RAS in the regulation of PI3-kinase [J]. Curr Top Microbiol Immunol, 2010, 346: 143-169. DOI: 10.1007/82_2010_56. 
45. Parsons CJ, Takashima M, Rippe RA. Molecular mechanisms of hepatic fibrogenesis [J]. J Gastroenterol Hepatol, 2007, 22 Suppl 1: S79-84. DOI: 10.1111/j.1440-1746.2006.04659.x.

46. Son G, Hines IN, Lindquist J, et al. Inhibition of phosphatidylinositol 3-kinase signaling in hepatic stellate cells blocks the progression of hepatic fibrosis [J]. Hepatology, 2009, 50(5): 1512-1523.

47. Gan D, Zhang W, Huang C, et al. Ursolic acid ameliorates CCl4-induced liver fibrosis through the NOXs/ROS pathway [J]. J Cell Physiol, 2018, 233(10): 6799-6813.

48. He W, Shi F, Zhou ZW, et al. A bioinformatic and mechanistic study elicits the antifibrotic effect of ursolic acid through the attenuation of oxidative stress with the involvement of ERK, PI3K/Akt, and p38 MAPK signaling pathways in human hepatic stellate cells and rat liver [J]. Drug Des Devel Ther, 2015, 9: 3989-4104. DOI: 10.2147/DDDT.S85426.

49. Ma JQ, Ding J, Zhang L, et al. Protective effects of ursolic acid in an experimental model of liver fibrosis through Nrf2/ARE pathway [J]. Clin Res Hepatol Gastroenterol, 2015, 39(2): 188-197.

50. Yang Y, Zhao Z, Liu Y, et al. Suppression of oxidative stress and improvement of liver functions in mice by ursolic acid via LKB1-AMP-activated protein kinase signaling [J]. J Gastroenterol Hepatol, 2015, 30(3): 609-618.

51. Xiang H, Han Y, Zhang Y, et al. A New Oleanolic Acid Derivative against CCI囚-Induced Hepatic Fibrosis in Rats [J]. Int J Mol Sci, 2017, 18(3): 553.

52. Yu SS, Chen B, Huang CK, et al. Ursolic acid suppresses TGF- $\beta 1$-induced quiescent HSC activation and transformation by inhibiting NADPH oxidase expression and Hedgehog signaling [J]. Exp Ther Med, 2017, 14(4): 3577-3582.

53. Kwon EY, Shin SK, Choi MS. Ursolic Acid Attenuates Hepatic Steatosis, Fibrosis, and Insulin Resistance by Modulating the Circadian Rhythm Pathway in Diet-Induced Obese Mice [J]. Nutrients, 2018, 10(11): 1719.

54. Wan S, Huang C, Wang A, et al. Ursolic acid improves the bacterial community mapping of the intestinal tract in liver fibrosis mice [J]. PeerJ, 2020, 8: e9050. DOI: 10.7717/peerj.9050.

55. Wan S, Luo F, Huang C, et al. Ursolic acid reverses liver fibrosis by inhibiting interactive NOX4/ROS and RhoA/ROCK1 signalling pathways [J]. Aging (Albany NY), 2020, 12(11): 10614-10632.

56. Lin X, Li Y, Zhang X, et al. Tormentic acid inhibits hepatic stellate cells activation via blocking $\mathrm{PI3K} / \mathrm{Akt} / \mathrm{mTOR}$ and NF-KB signalling pathways [J]. Cell Biochem Funct, 2021, 39(1): 77-87.

57. Haraguchi H, Ishikawa H, Kubo I. Antioxidative action of diterpenoids from Podocarpus nagi [J]. Planta Med, 1997, 63(3): 213-215. 
58. Liao YJ, Bai HY, Li ZH, et al. Longikaurin A, a natural ent-kaurane, induces G2/M phase arrest via downregulation of Skp2 and apoptosis induction through ROS/JNK/c-Jun pathway in hepatocellular carcinoma cells [J]. Cell Death Dis, 2014, 5(3): e1137.

59. Qin S, Chen SH, Guo YW, et al. Diterpenoids of Isodon macrophylla [J]. Helvetica Chimica Acta, 2007, 90(10):2041-2046.

60. Duan H, Wang GC, Khan GJ, et al. Identification and characterization of potential antioxidant components in Isodon amethystoides (Benth.) Hara tea leaves by UPLC-LTQ-Orbitrap-MS [J]. Food Chem Toxicol, 2021, 148: 111961. DOI: 10.1016/j.fct.2020.111961.

61. Thao TTP, Lieu NT, Ninh PT, et al. Study on the chemical constituents of Dacrydium elatum and their cytotoxic activity [J]. Zeitschrift für Naturforschung B, 2019, 74(2). DOI囚10.1515/znb-2018-0214.

\section{Figures}


A

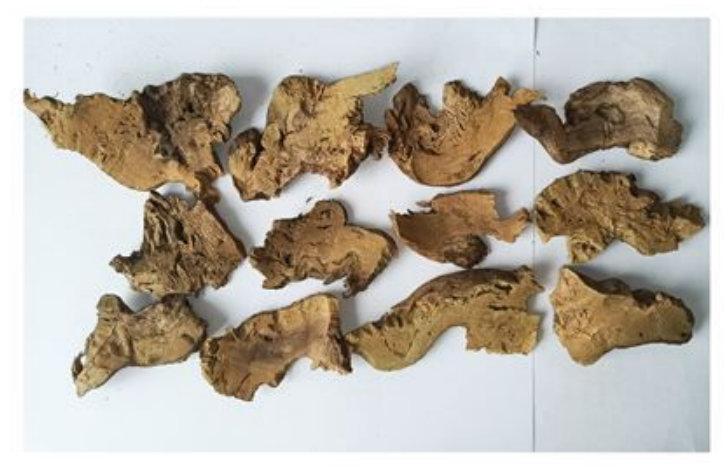

B

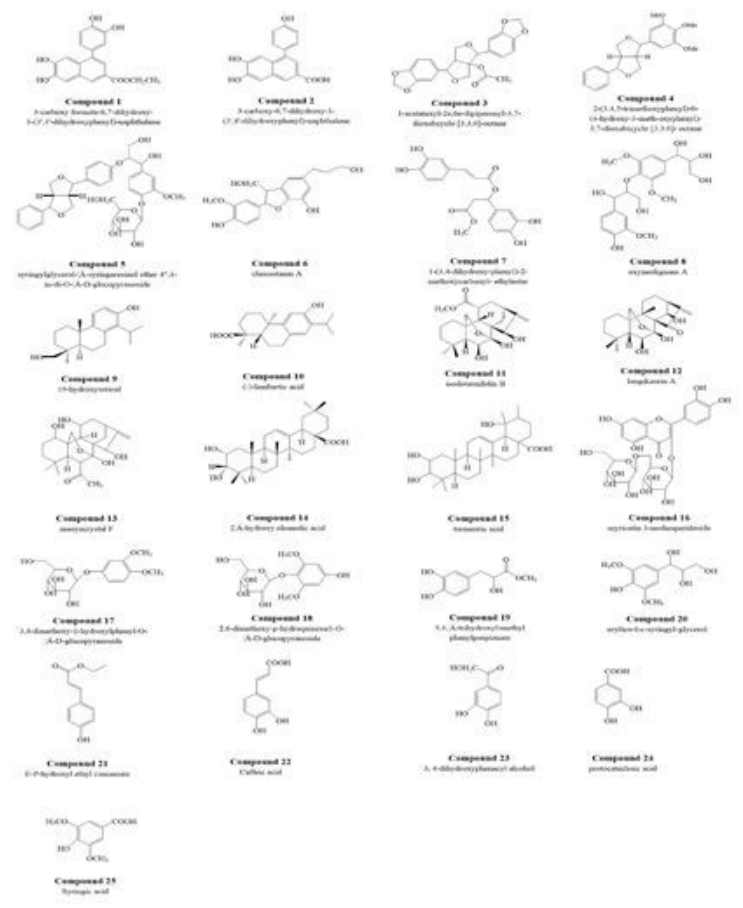

$\mathrm{C}$
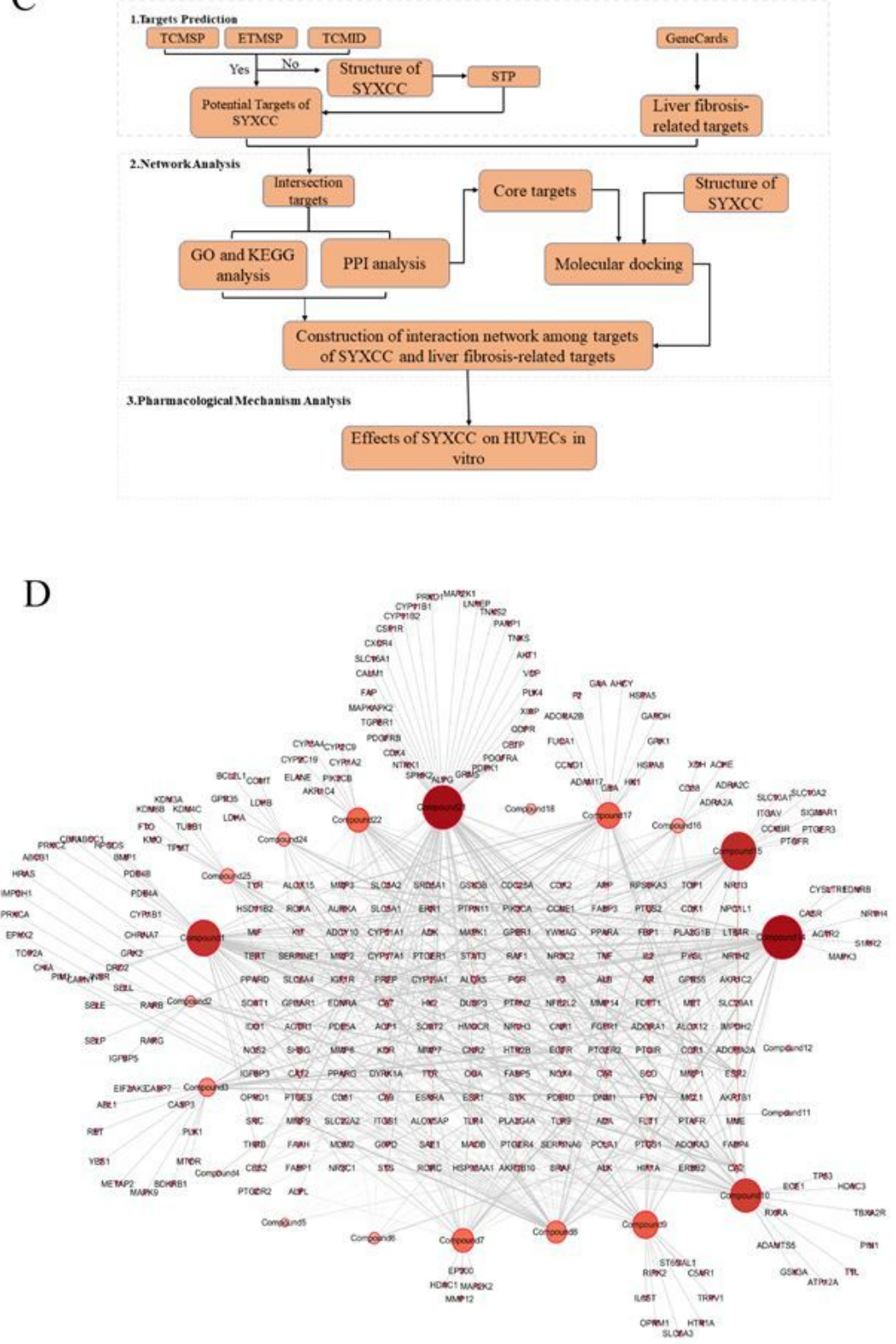

Figure 1

Workflow of the network pharmacology approach to analysis common target proteins of SYXCC ingredients and liver fibrosis. (A) The picture shows dried rhizomes of SYXCC. (B) Twenty-five chemical constituents of SYXCC were obtained through literature search. (C) Network pharmacology workflow diagram. (D) Interaction network between SYXCC and liver fibrosis target protein. The dots indicate ingredient of SYXCC, the darker and larger dots, the greater amount of ingredient with liver fibrosis target proteins. Triangle represents liver fibrosis target protein. The larger triangle size is, the deeper color is, indicating that the protein is more closely related to the ingredient of SYXCC. (Note: SYXCC represents Isodon ternifolius) 
A

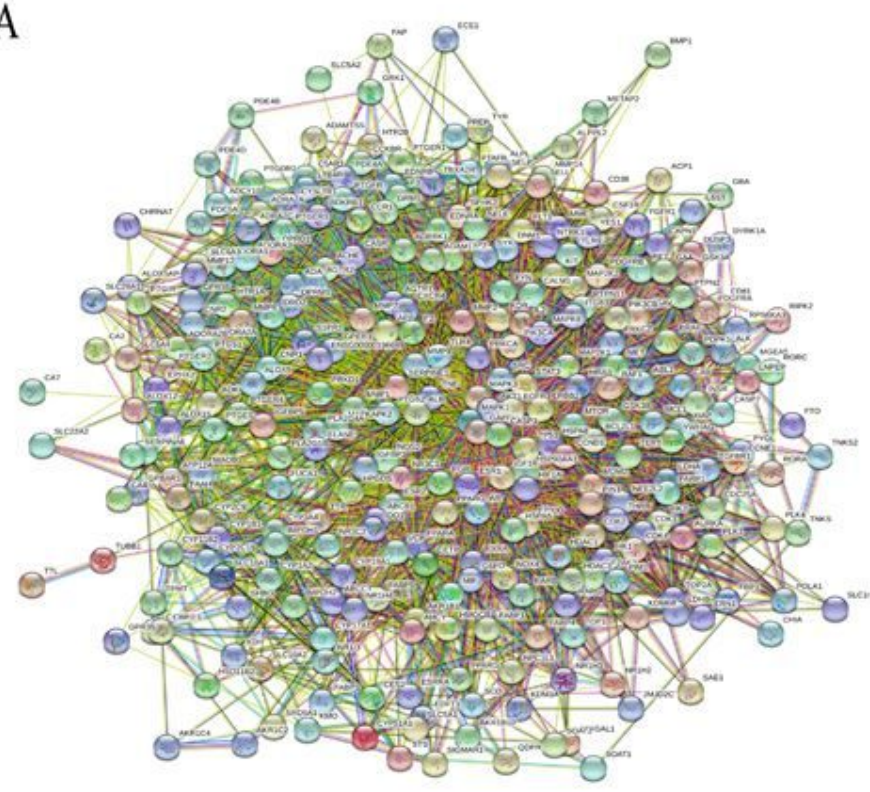

B

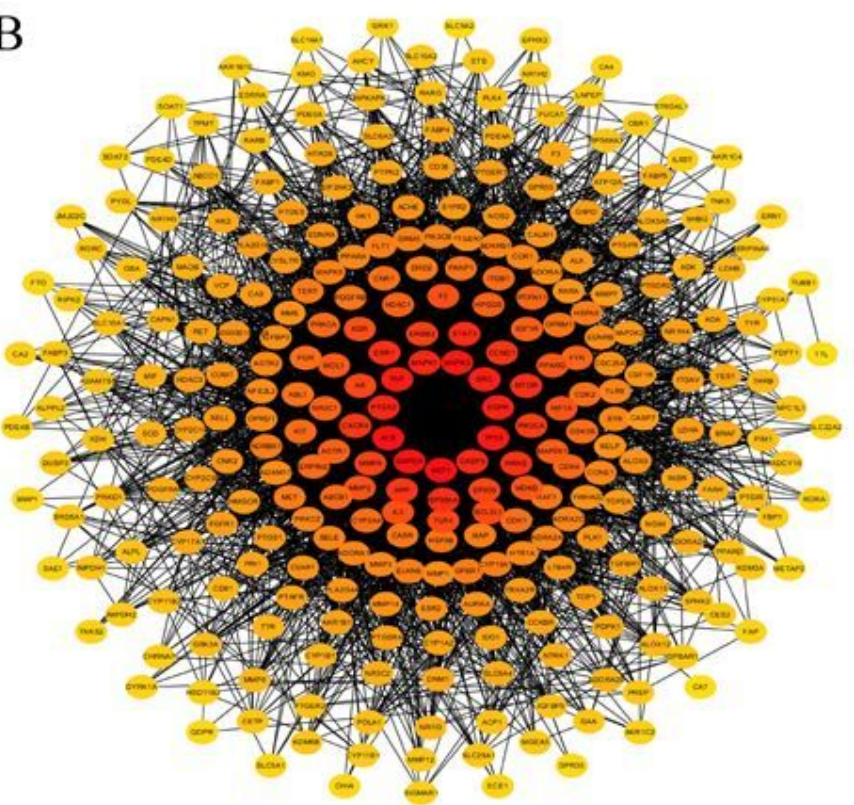

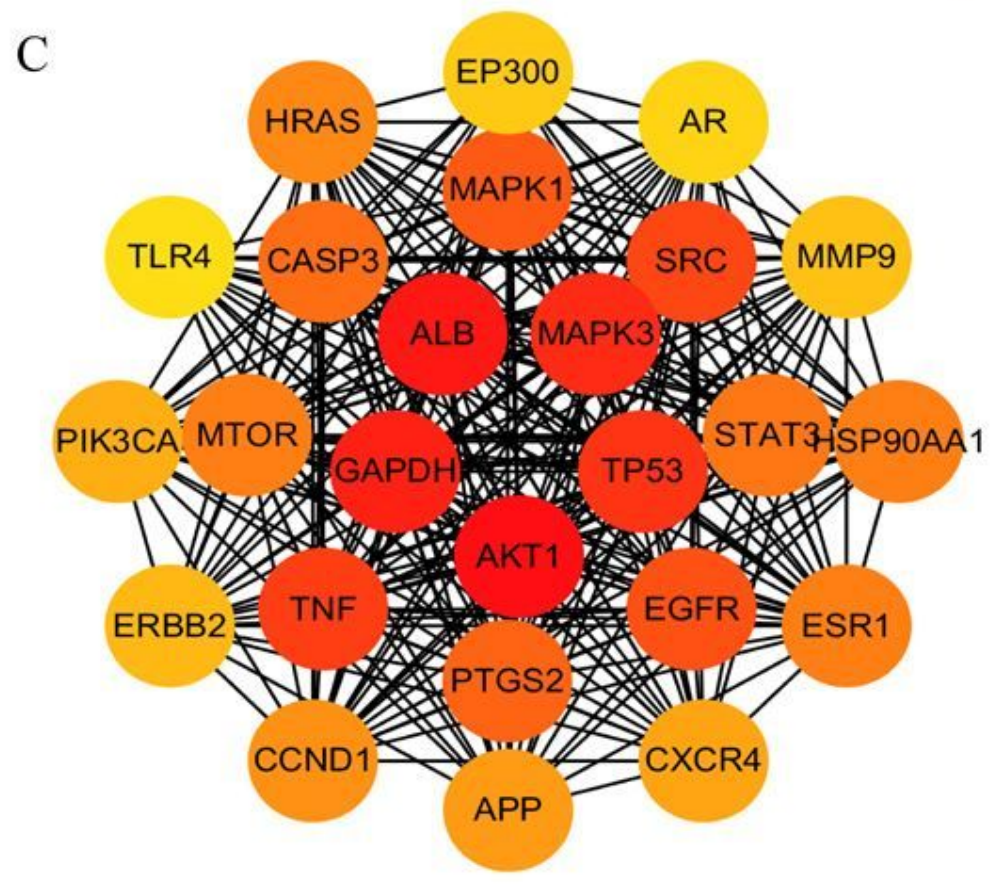

Figure 2

Protein-protein interaction network (PPI) of common target proteins of SYXCC and liver fibrosis. (A) The original PPI network graph derived from STRING data has 298 nodes, 626 edges, and average node degree value is 29.6. (B) The initial PPI network map was visualized by cytoscape software, the darker colour, the greater number of ineractions with liver fibrosis network target proteins. (C) The top 25 target protein interaction network graphs were obtained by topological network structure analysis. The deeper the color was, the more target proteins interacted with liver fibrosis network. 

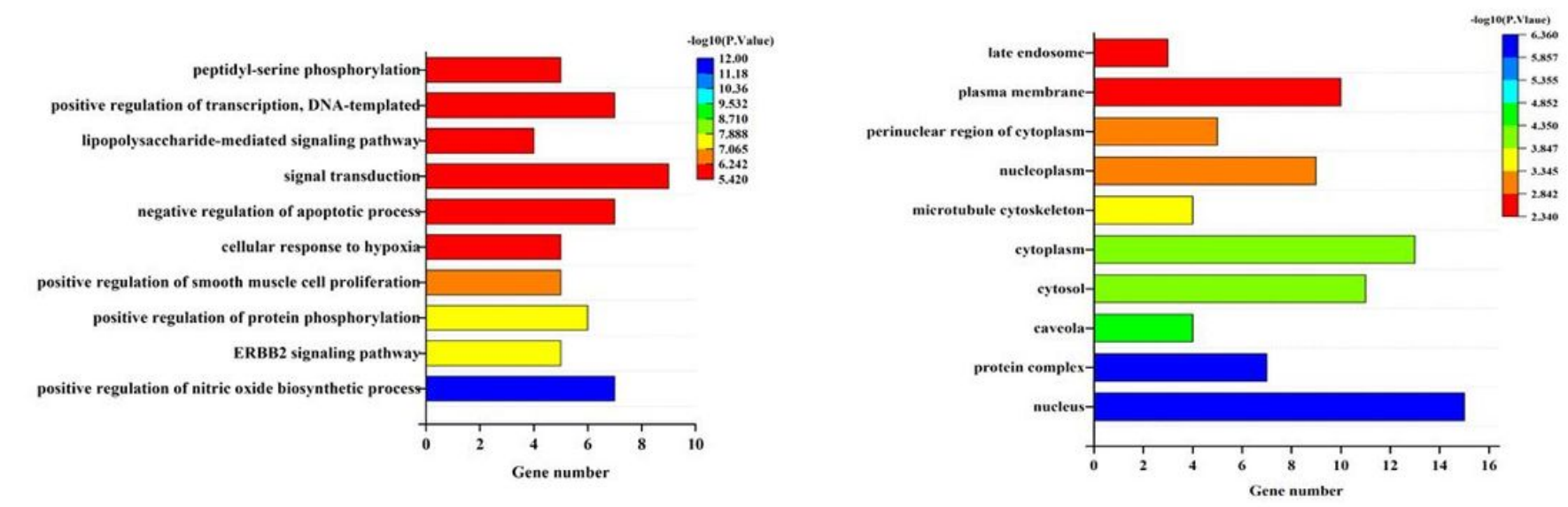

B

(Molecular Function, MF)

D

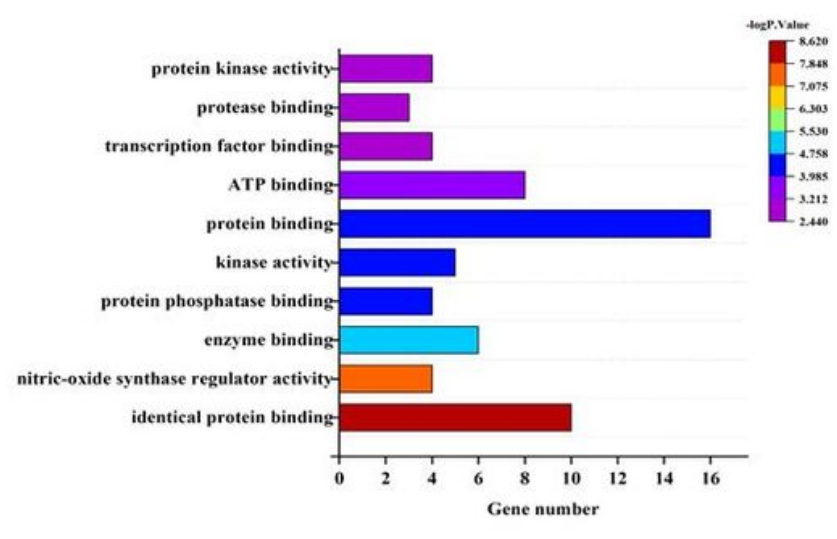

\section{Figure 3}

GO and KEGG enrichment analysis. GO enrichment analysis includes (A) The top 10 terms of biological process (BP). (B) The top 10 terms of Molecular function (MF). (C) The top 10 terms of cellular component (CC). KEGG pathway enrichment analysis (D) The top 25 terms of signaling pathways,

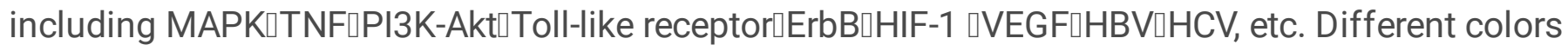
represent different adjusted p-values, and dot size represents numbers of genes enriched in each signaling pathway. 


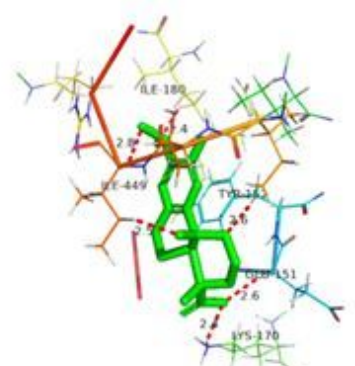

(a) Ala1-(-)-Lambertic acid (C10) Mnimues bialeg energat -9.05

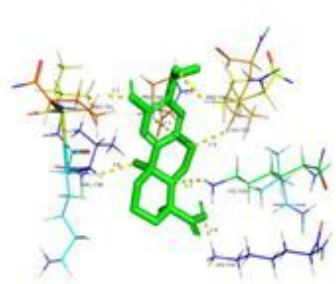

(b) EGFR-(-)-Lamberbic acd (C10) Minimum binding energy: -6.51

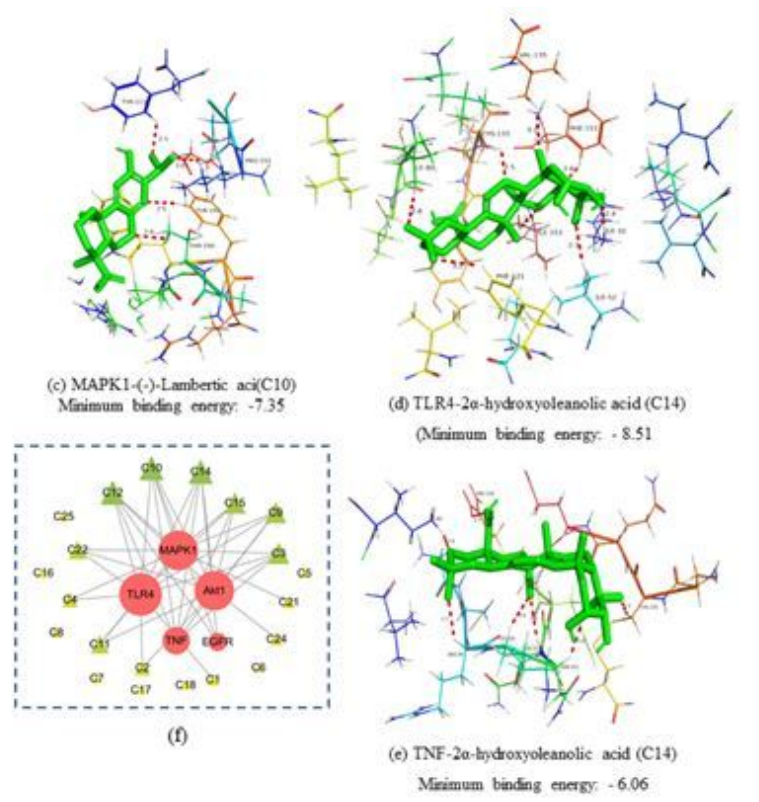

B

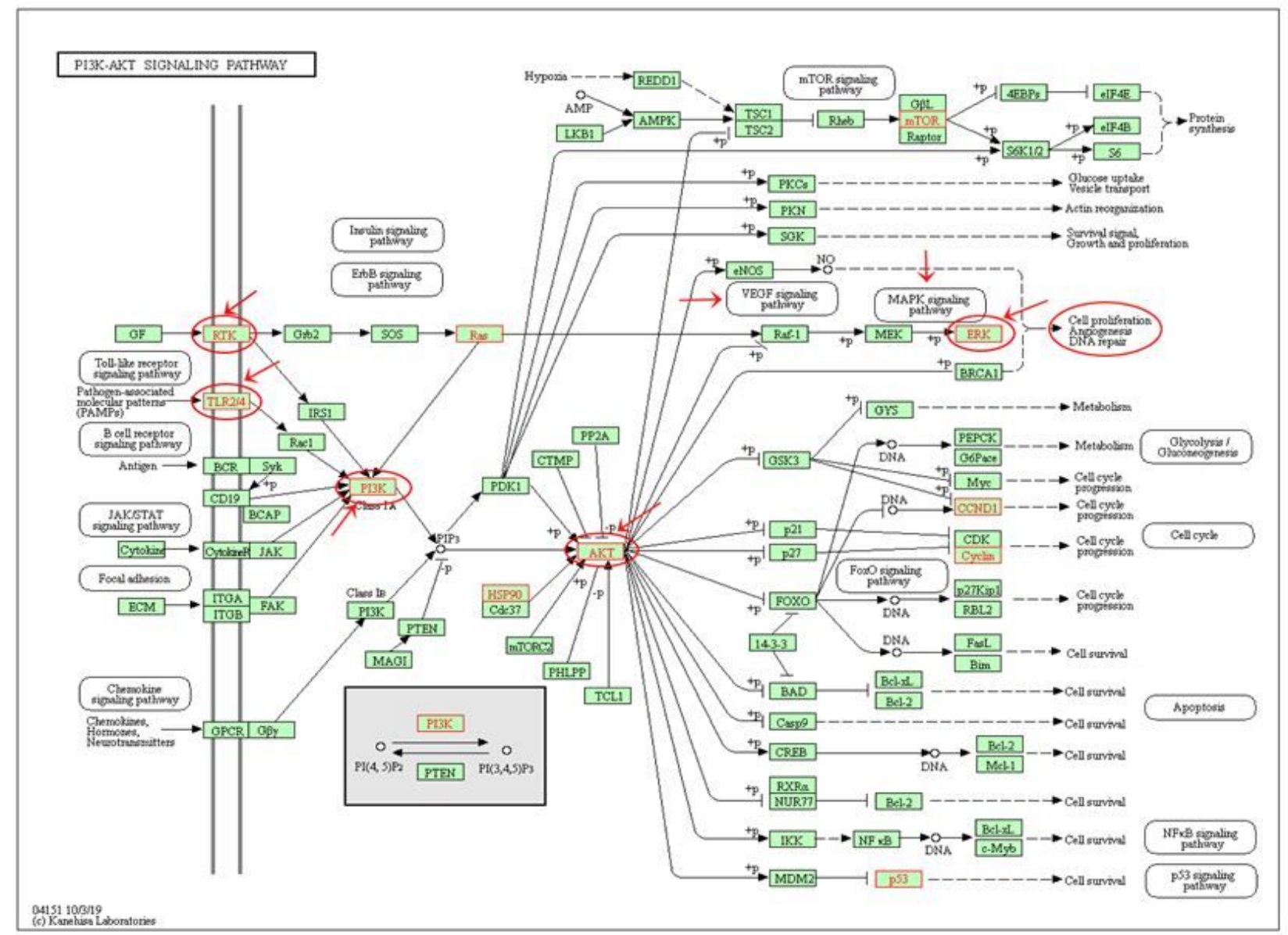

\section{Figure 4}

Molecular docking. (A) (a, b, c, d) Docking model with the lowest binding energy between SYXCC ingredient and core targets of TNF, Akt, MAPK1, EGFR and TLR4. (f) Binding energy $\mathbb{-}-5$ was used as evaluation criterion for drug docking with core targets. Compounds $10,12,14$ and 15 could bind to multiple core targets, and TLR4, MAPK1 and Akt1 could bind to ingredients of SYXCC most. Red dots represent core targets, and the larger the dots, the more ingredients are combined. Green triangle 
represents components that could dock with core targets, and size of dots represents numbers of docking with core targets. Yellow triangle represents components that fail to dock with core targets. (B) Map of PI3K-AKT signaling pathway derived from KEGG URL (https://www.genome.jp/kegg/). Core targets TNF, Akt1, MAPK1, EGFR and TLR4 were involved in PI3K-AKT signaling pathway, regulating cell proliferation, DNA damage and angiogenesis.

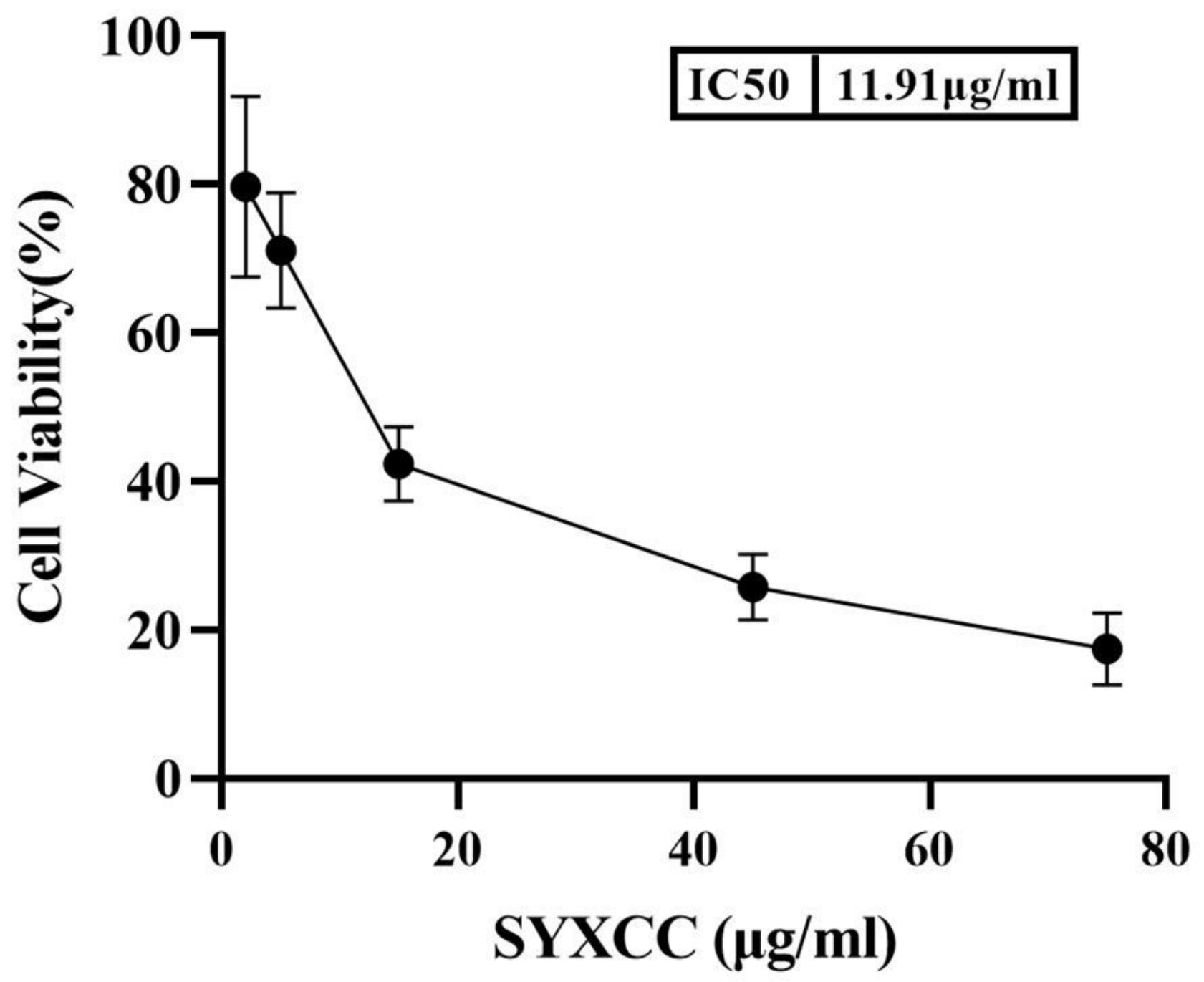

Figure 5

Effect of different concentration of SYXCC on HUVECs activity. (Note: SYXCC represents Isodon ternifolius) 
A

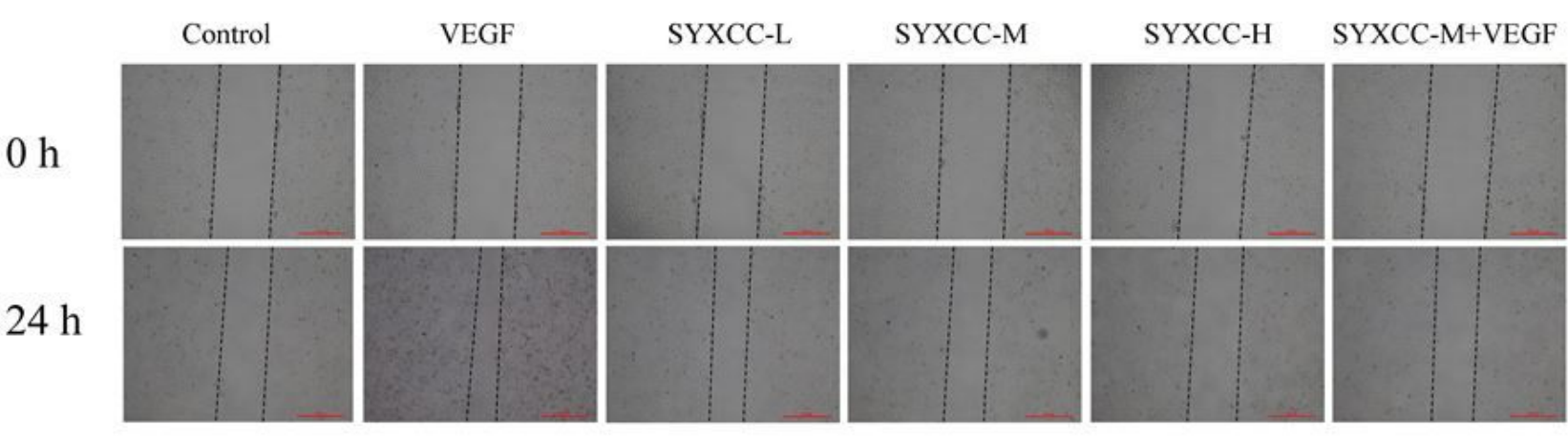

$\mathrm{B}$
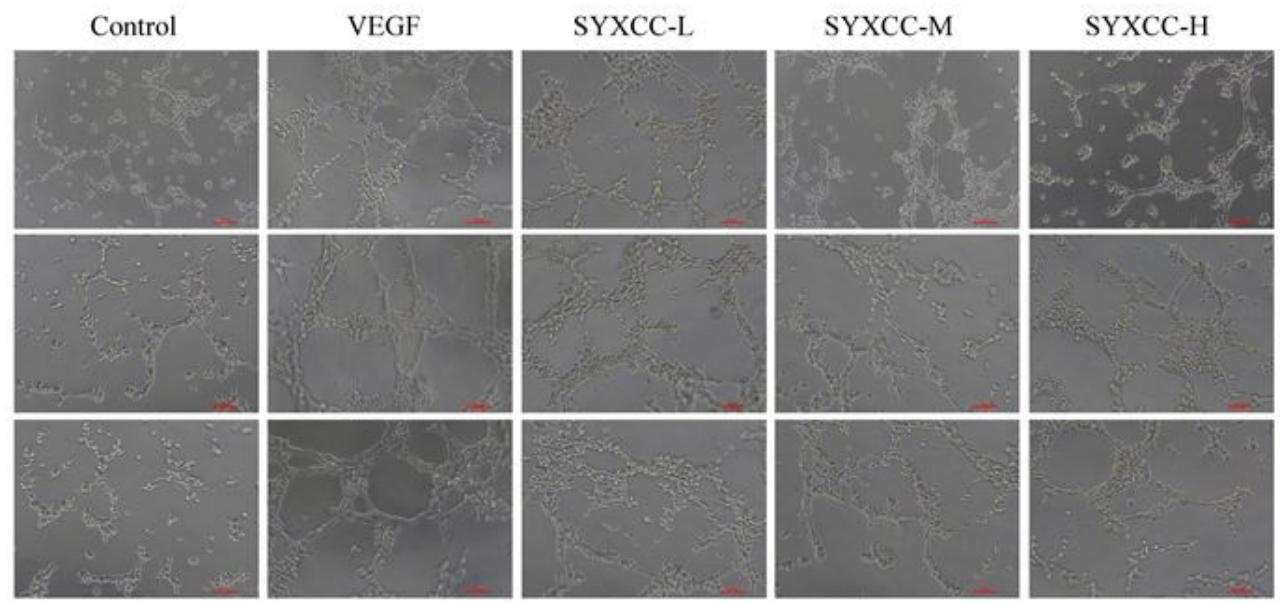

SYXCC-M+VEGF
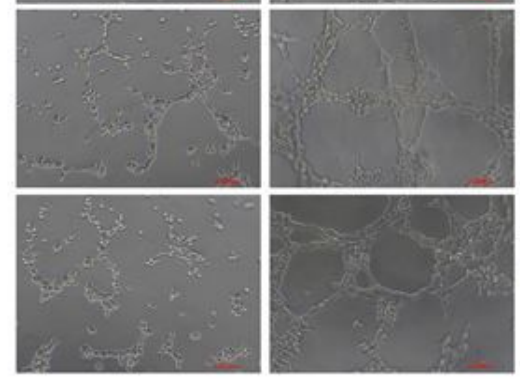

$\mathrm{C}$

$\mathrm{D}$
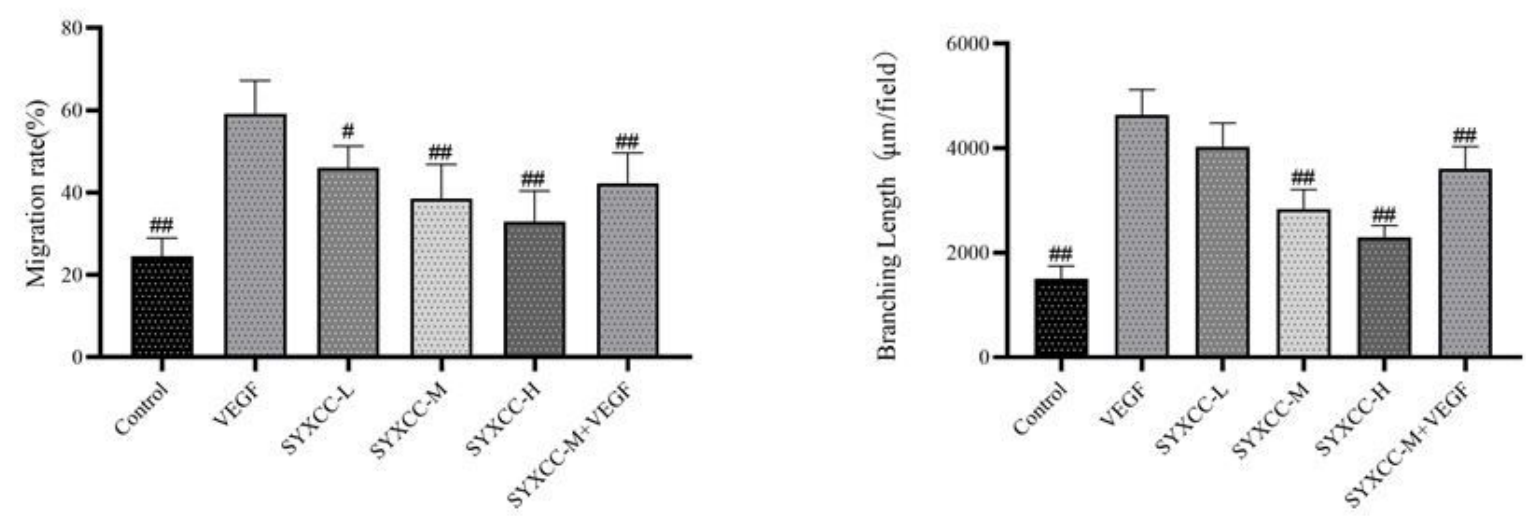

\section{Figure 6}

Effect of SYXCC extract on cell migration ability and tube formation. In vitro, human umbilical vein endothelial cells were treated with blank control, VEGF $(10 \mathrm{ng} / \mathrm{ml})$, low-dose SYXCC $(10 \mu \mathrm{g} / \mathrm{ml})$, mediumdose SYXCC $(20 \mu \mathrm{g} / \mathrm{ml})$, high-dose SYXCC $(40 \mu \mathrm{g} / \mathrm{ml})$, or medium-dose SYXCC $(10 \mathrm{ng} / \mathrm{ml})+$ VEGF $(10 \mathrm{ng} / \mathrm{ml})$. (A) Scratch healing assays. (B) Tube formation assays $(n=3)$. (C) Quantitative analysis of scratch healing assays $(n=3)$. (D) Quantitative analysis of tube forming assays $(n=3)$. All data are expressed as mean \pm SD. \#P $<0.05$ (VS VEGF group), \# \# P< 0.01 (VS VEGF group). (SYXCC: SYXCC, L: low dose, M: medium dose, $\mathrm{H}$ : hight dose) 
A

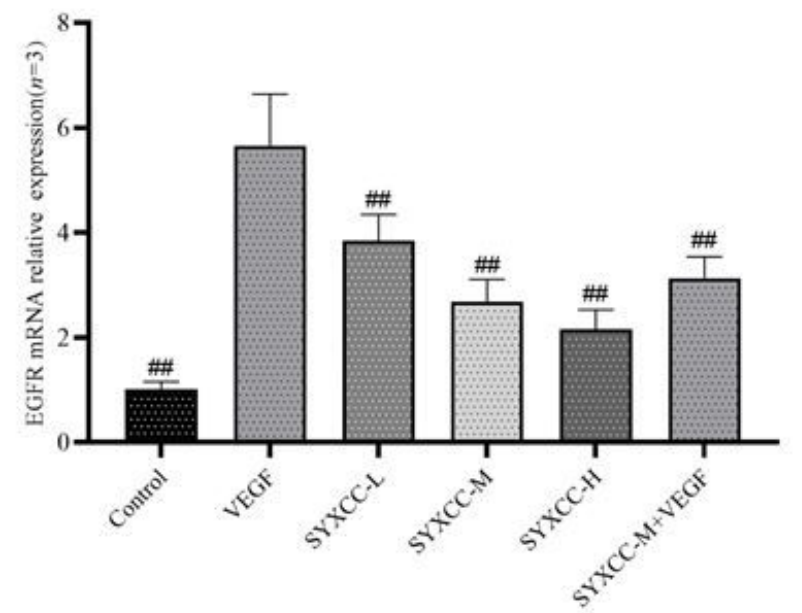

B

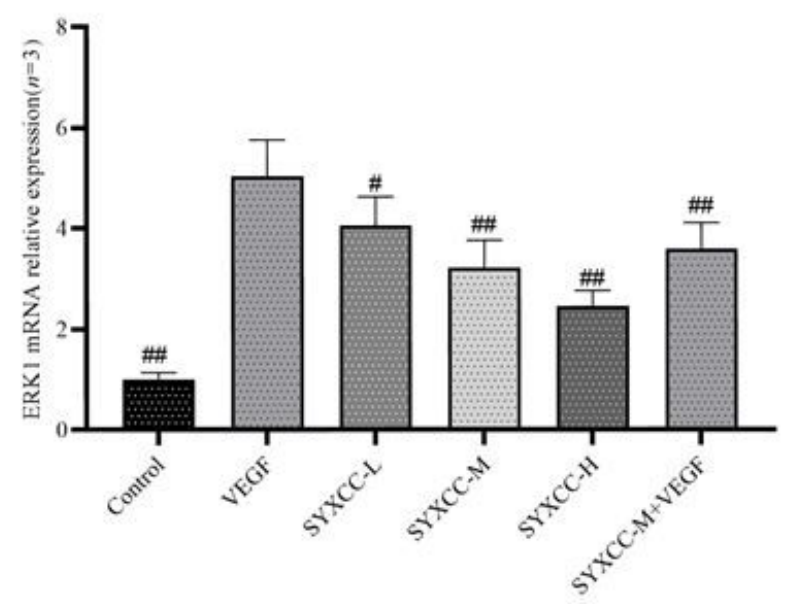

$\mathrm{C}$

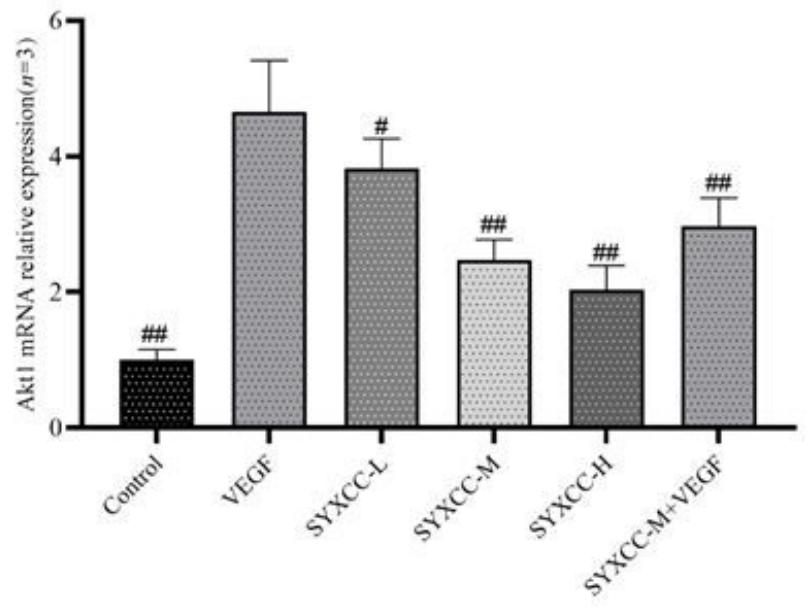

D

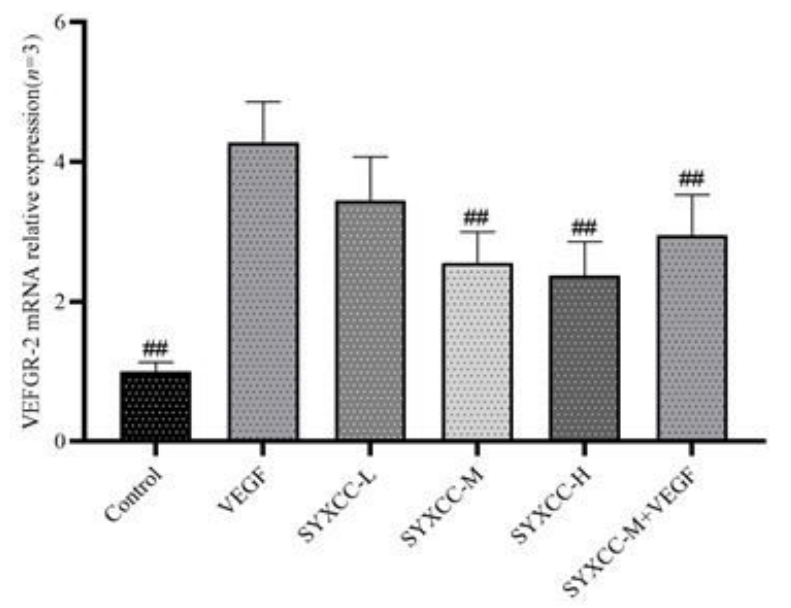

E

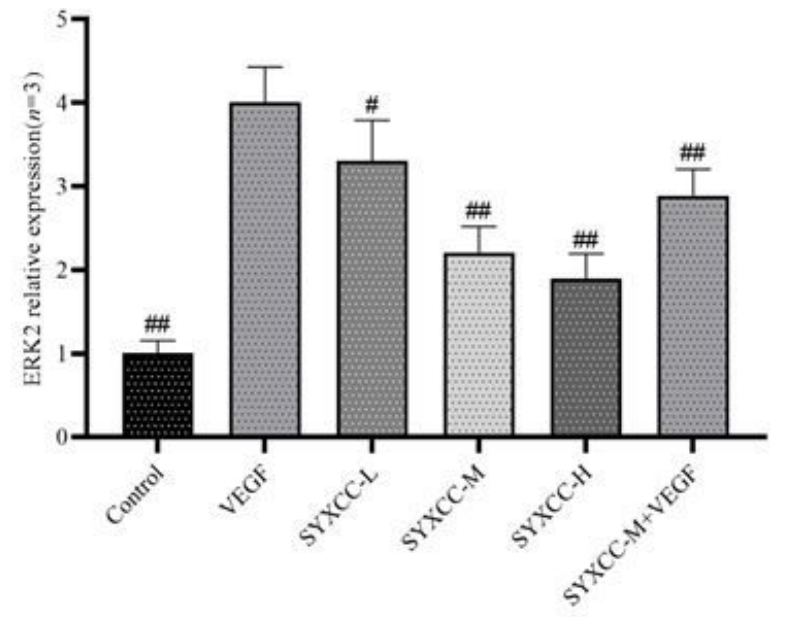

\section{Figure 7}

Effect of SYXCC on expression of core target genes. In vitro, human umbilical vein endothelial cells were treated with blank control, VEGF $(10 \mathrm{ng} / \mathrm{ml})$, low dose SYXCC $(10 \mu \mathrm{g} / \mathrm{ml})$, medium dose SYXCC $(20 \mu \mathrm{g} / \mathrm{ml})$, high dose SYXCC $(40 \mu \mathrm{g} / \mathrm{ml})$ or medium dose + VEGF (10ng/ml). (A, B, C, D, E) EGFR, ERK1, Akt1, VEFGR2, ERK2 mRNA expression was analyzed by PCR ( $n=3)$. (SYXCC: SYXCC, L: low dose, M: medium dose, H: hight dose) 


\section{Supplementary Files}

This is a list of supplementary files associated with this preprint. Click to download.

- AKT1autodocktest.rar

- EGFRautodocktext.rar

- MAPK1autodocktest.rar

- STRINGdata.rar

- TLR4autodocktest.rar

- TNFautodocktest.rar

- swisstargetpreditionresult.rar 